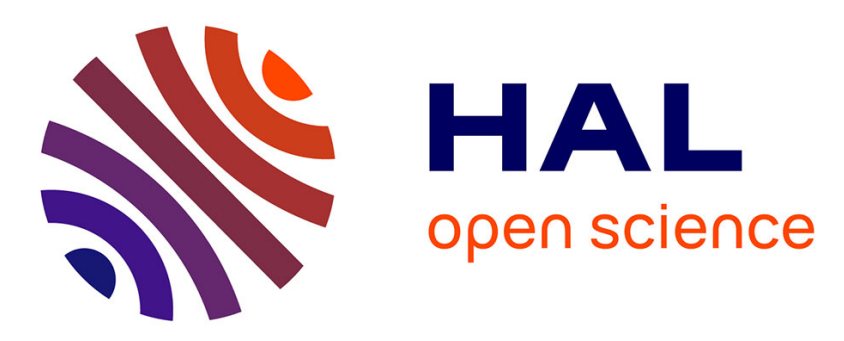

\title{
Decision process to manage useful life of multi-stacks fuel cell systems under service constraint
}

Nathalie Herr, Jean-Marc Nicod, Christophe Varnier, Louise Jardin, Antonella Sorrentino, Daniel Hissel, Marie-Cécile Péra

\section{- To cite this version:}

Nathalie Herr, Jean-Marc Nicod, Christophe Varnier, Louise Jardin, Antonella Sorrentino, et al.. Decision process to manage useful life of multi-stacks fuel cell systems under service constraint. Renewable Energy, 2017, 105, pp.590 - 600. hal-02130997

\section{HAL Id: hal-02130997 https://hal.science/hal-02130997}

Submitted on 16 May 2019

HAL is a multi-disciplinary open access archive for the deposit and dissemination of scientific research documents, whether they are published or not. The documents may come from teaching and research institutions in France or abroad, or from public or private research centers.
L'archive ouverte pluridisciplinaire HAL, est destinée au dépôt et à la diffusion de documents scientifiques de niveau recherche, publiés ou non, émanant des établissements d'enseignement et de recherche français ou étrangers, des laboratoires publics ou privés. 


\title{
Decision process to manage useful life of multi-stacks fuel cell systems under service constraint *
}

\author{
Nathalie Herr ${ }^{\ddagger}$, Jean-Marc Nicod ${ }^{\ddagger}$, Christophe Varnier \\ Louise Jardin ${ }^{\ddagger}$, Antonella Sorrentino ${ }^{\ddagger}$, \\ Daniel Hissel ${ }^{\S \uparrow}$ and Marie-Cécile Péra ${ }^{\S \uparrow}$ \\ ${ }^{\ddagger}$ FEMTO-ST/AS2M, Université Bourgogne Franche-Comté/ \\ UFC/CNRS/ENSMM/UTBM, Besançon, France \\ Emails: [nathalie.herr/jean-marc.nicod/christophe.varnier]@femto-st.fr \\ ${ }^{\S}$ FEMTO-ST/Energy, Université Bourgogne Franche-Comté/ \\ UFC/CNRS/ENSMM/UTBM, Belfort, France \\ Emails: [daniel.hissel/marie-cecile.pera] @femto-st.fr \\ FCLAB Research Federation, FR CNRS 3539, Belfort, France
}

\begin{abstract}
A management of multi-stacks fuel cell systems is proposed to extend systems useful life in a Prognostics and Health Management (PHM) framework. The problem consists in selecting at each time which fuel cell stacks have to run and which output power has to be chosen for each of them to satisfy a load demand as long as possible. Multi-stacks fuel cell system useful life depends not only on each stack useful life, but also on both the schedule and the operating conditions settings that define the contribution of each stack over time. As the impact of variable operating conditions on fuel cell lifetime is not well-known, a simplified representation of fuel cell behavior under wear and tear is used to estimate the available outputs over time and their associated Remaining Useful Lives $(R U L)$. This health state prognostics model is configured to suit to Proton-Exchange Membrane Fuel Cells (PEMFC) specific characteristics. The proposed scheduling process makes use of an optimal approach based on a Mixed Integer Linear Program (MILP). Efficiency of the associated commitment strategy is assessed by comparison with basic intuitive strategies, considering constant and piecewise constant load demand profiles.
\end{abstract}

Keywords: PHM, Decision making, Scheduling, Useful Life, Optimization

${ }^{*}$ This is the author's version of an article published in Renewable Energy 105C (2017) 


\section{Introduction and related work}

Fuel cells appear to be of growing interest for power conversion [1]. This technology offers indeed a potential alternative to conventional power systems and is involved in many applications. Fuel cells are for instance increasingly being used as backup systems in hybrid power supply systems composed of renewable energy sources [2], such as solar and wind generator. In fact, renewable energy sources being intermittent by nature, they need to be combined with other systems which can generate power on demand. In this context, fuel cells are only used occasionally, during periods when the energy produced by renewable sources is not enough to meet the load demand. Azcarate et al. [3] developed for instance a simulation model for a wind- $\mathrm{H}_{2}$ energy system and proposed dynamic management policies based on the conversion of electricity into hydrogen and the use of the stored hydrogen to produce electricity during demand peaks using a hydrogen fuel cell. Bigdeli [4] proposed different optimal techniques for the management of a hybrid electric power generation system that consists of a photovoltaic array as primary energy source and of a fuel cell and a battery as backup units. Load sharing among the available resources to achieve optimal performance is addressed using several optimization approaches (imperialist competitive algorithm, particle swarm optimization, quantum behaved swarm optimization, ant colony optimization and Cuckoo optimization algorithm). Studies proposed in the literature considering fuel cells technology in such power supply systems mainly focused on the evaluation of their performance on life cycle cost, optimal sizing and hybridazation, rather than optimal control [2]. For this latter purpose, Sichilalu et al. [2] developed an optimal control strategy model for an integrated grid system considering renewable energy sources (wind and photovoltaic) and fuel cells. The proposed control strategy avoids using the fuel cells at all times: they are used only during peak periods or when the main power generation system is completely unavailable.

Combined with batteries, fuel cells are also being used in stand-alone applications, in which they mainly serve as indirect storage of energy and as stable power sources. Lopez et al. [5] developed for instance two genetic algorithms to find an optimal configuration of the components involved in a stand-alone renewable energy hybrid system with hydrogen storage (power conversion being performed by a fuel cell). First proposed genetic algorithm searches for possible component configurations, whereas second one optimizes the strategy for each of the configurations. Abadlia et al. [6] considered the power management of a renewable energy source associated to an energy storage system composed of a proton exchange membrane fuel cell (PEMFC) and batteries in a photovoltaic system used in stand-alone. They proposed a fuzzy logic power management strategy to ensure a good management of the power flow, which maximizes the production of hydrogen and controls the charge/discharge mode operation of the battery. In these stand-alone applications, as well as in the hybrid power supply systems introduced previously, the main challenge is to properly control the load power sharing among the main and the secondary power sources in order to comply with the availability of the different energy sources considered and with the variability of the load demand.

Fuel cells are also used as primary sources and coupled with batteries to provide the necessary power for transportation applications. For such applications, fuel cell systems are considered as the best energy sources that reduce 
fuel consumption and $\mathrm{CO}_{2}$ emissions [7]. Some studies have considered the use of fuel cells for electric vehicles. Aouzellag et al. [8] proposed a control strategy to manage the power distribution among two energy sources (PEM fuel cell and battery) for fuel cell hybrid electric vehicles. Neffati et al. [9] tackled also the power management for a hybrid full electric vehicle with a fuel cell system as main energy source and a super-capacitor as storage element. The considered objective was to minimize the overall cost of hydrogen consumption for a given power demand. This was performed by optimizing the power distribution on the two sources considering availability, performance and state of charge constraints. An off-line optimization strategy for the energy management has been proposed, in which dynamic programming has been used to provide a benchmark regarding the maximum potential fuel savings. An on-line strategy based on fuzzy switching of fuzzy rules has also been proposed for a real-time energy management taking into account the evolution over time of the state of charge of the storage element.

Contrary to a vast majority of contributions in the literature addressing the use of fuel cell systems, such as those previously introduced, fuel cells are considered in this article as the unique power source. All the auxiliary functions necessary to guaranty a good functioning of fuel cells are moreover not considered in this study and are supposed to be well managed by auxiliaries, independently from the addressed decision process. For instance, the storage of hydrogen or the management of the chemical reactions that lead to power conversion are out of the scope of the proposed contribution, in which fuel cells associated to their auxiliaries are seen as black boxes that deliver a certain power output. In order to reach suitable power outputs, systems composed of several fuel cell stacks are considered. Each stack is supposed to be independent but the multi-stacks system has to globally deliver a given power output based on a need of energy. At each time, this global output is determined by the sum of each output of the stacks that are currently running. The scheduling of such systems is addressed, with the maximization of their global useful life as objective. In fact, the increase of fuel cells lifetime and reliability has been highlighted by Borup et al. [10] to be an important challenge. Durability of fuel cells is indeed not consistent with most applications. Then, we propose to contribute to this challenge by defining an original management of a set of fuel cells stacks. Our purpose is not to improve each fuel cell lifetime, but to propose a way to use several fuel cells in parallel in an appropriate manner so as to globally increase the whole system durability.

Considering a global needed power output, the multi-stacks system useful life depends not only on each stack useful life, but also on both the schedule and the operating condition settings that define the contribution of each stack over time. Indeed, operating conditions have been shown by Borup et al. [10] to have an impact on each fuel cell output and on their durability. The same statement applies to batteries. The maximization of a battery charge used while constraining the probability of a shut off in flight has been studied by Saha et al. [11] for electric unmanned aerial vehicles. Prognostics is used to predict remaining battery life. Advantage is taken of this information to optimize mission plans without exceeding the available battery charge. In a same way, fuel cell available outputs and their associated lifetime can be determined at each time by a Prognostics and Health Management (PHM) process based on both the past and the future operating conditions [1]. Steps involved in a $P H M$ process are 
depicted in Figure 1. It has been pointed out by Jouin et al. [1] that researches in PHM dealing with fuel cells have been mainly focused on data acquisition and data processing. Less attention has been paid to condition assessment and diagnostics and few works addressed prognostics. Papers taking into account decision making are even scarcer. In those papers, the decision part deals furthermore only with corrective actions (see [12] and [13]), which tend to master fuel cell operating conditions through the control of physical parameters. This real-time control operates at timescales from nanoseconds to seconds. In the decision process involved in this paper, larger timescales (hours to weeks) are taken into consideration. Knowing this, the purpose is to select at each time which fuel cell stacks have to run and which power output has to be chosen for each of them to satisfy the load demand as long as possible.

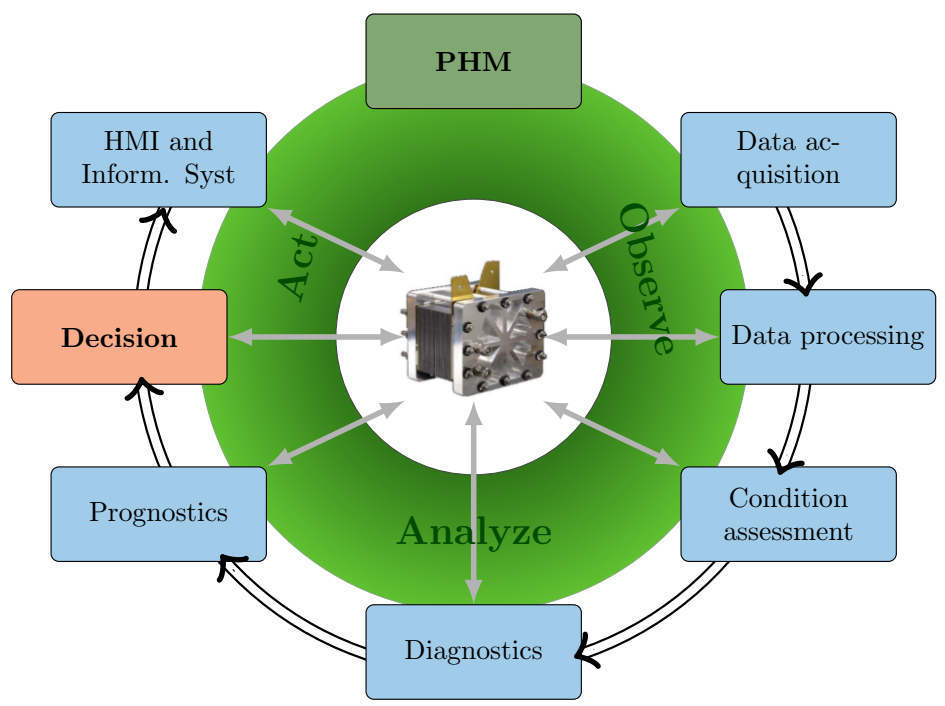

Figure 1: $P H M$ process

A similar problem has been addressed in [14] and [15] considering machines able to provide a discrete number of throughputs. It has been shown that a schedule can be adapted to machines health state by considering prognostics results in the form of $R U L$. The associated decision problem being NPcomplete [15], optimal solutions can be found in limited time for small size problem instances only, considering very few machines, few different throughput values and short production horizons. Many heuristics have been provided to cope with the problem of maximizing a platform useful life under service constraint for larger instances. Efficiency of these heuristics have been assessed through exhaustive simulations, but the considered approach cannot be straightforwardly applied to fuel cell systems. In order to suit to fuel cells, one has indeed to consider outputs that can vary continuously and take any value within a given interval. Furthermore, due to the aging, each maximal output is supposed to be decreasing with time. A scheduling process taking into account these fuel cells specific features is proposed in this article. As the impact of variable operating conditions on fuel cell lifetime is up to now not well-known, a simplified trend depicting the wear and tear behavior of fuel cells stacks is 
used to determine at each time the range of available power outputs and their associated RUL.

The application framework and the decision problem are described in Section 2. A simplified representation of fuel cell wear and tear behavior is proposed in Section 3. The proposed health state prognostics model is detailed and compliance with fuel cell characteristics is assessed. A commitment strategy making use of an optimal approach based on a Mixed Integer Linear Program (MILP) is then detailed in Section 4. This strategy can be used to cope with load demands that are either constant over time or piecewise constant during the production horizon. Simulation results showing the efficiency of the proposed strategy are provided in Section 5 and the work is concluded in Section 6 .

\section{Problem statement}

\subsection{Application framework}

The application addressed in this paper is based on a multi-stacks fuel cell system composed of a set $\mathscr{M}$ of $m$ Proton-Exchange Membrane Fuel Cell (PEMFC) stacks $M_{j}(1 \leqslant j \leqslant m)$, with $\mathscr{M}=\left\{M_{1}, \ldots, M_{j}, \ldots, M_{m}\right\}$. Such a system is supposed to meet energy requirements for domestic usage in a stationary power generation framework, also known as micro combined heat and power (microCHP). Cyclic variation of the load demand based on domestic needs in energy is then taken into account. In this context, following assumptions are made on the considered multi-stacks fuel cell system:

- each stack $M_{j}$, composed of many individual connected single cells, is supposed to be able to deliver global power outputs $P_{j}$ that can vary continuously within a given power output interval. While the minimal output is supposed to be constant over time, the maximal one decreases with time when the stack is used. Then, the range of available outputs depends on the time $t: 0 \leqslant P \min _{j} \leqslant P_{j}(t) \leqslant P \max _{j}(t)$;

- the management of auxiliary systems is not considered in this study:

- auxiliary systems are supposed to be configured and managed separately so that fuel cell stacks are able to provide the needed power outputs while being kept in operating conditions which do not accelerate the cells wear and tear ;

- all the stacks are supposed to be always supplied with all reactants required for fuel cell internal chemical reactions leading to the power conversion ;

- fuel cell stacks can be used simultaneously and independently from each other ;

- at each time, the global system outcome Ptot is supposed to be the sum of each stack power contribution ;

- during the whole production horizon $\mathcal{H}$, the global output $P$ tot has to reach a given load demand $\sigma(t)$. In the stationary power generation framework considered, this demand is supposed to be piecewise constant over time ;

- overproduction is authorized, but is lost. In fact, storage is not considered in this study ;

- all the fuel cell stacks are not supposed to be in use at each time if a subset 
of them is enough to reach the demand. Fuel cells suffering from wear and tear, some stacks can also be not available if their end of life $(E O L)$ has been reached ;

- no $R U L$ overrun is allowed ;

- stop-and-start of stacks are not allowed. Stopping and restarting a fuel cell can indeed induce considerable damage [10]. Then, when a stack has been started up, it is used until its end of life.

This last assumption is very restrictive. Change of output is however still authorized during the stacks lifetimes. This leads to a decision problem, detailed in next section.

\subsection{Decision problem}

Considering the assumptions detailed in previous section, the point is to manage the fuel cell system by defining at each time which fuel cell stacks have to be used and their contribution to the global output so as to reach the load demand $\sigma(t)$ as long as possible. In fact, instant needed power values have to be determined for each stack and provided to the control system over time during the whole production horizon. One way to tackle the problem consists in discretizing the time into periods $\Delta T$. This approach is not so far from realistic constraints, since one can imagine that one period could be one hour, one day or one week in a real case. The production horizon $\mathcal{H}$ can then be expressed as follows: $\mathcal{H}=K \times \Delta T$, with $K$ the number of periods for which the load demand $\sigma(t)$ is reached. Periods are supposed to be very small compared to $R U L$ values $\left(\Delta T \ll R U L_{j} \forall j=1, \ldots, m\right)$.

Two cases are considered in this study. The demand can be constant during the whole production horizon and is then called $\sigma$. A cyclic variation of the demand can also be considered for a stationary application, for instance for domestic usage in a micro-CHP context. In this case, the demand is supposed to be piecewise constant and is denoted $\sigma(t)=\sigma_{k}$, with $\sigma_{k}$ a constant value within the period $k$ such that $(k-1) \Delta T<t \leqslant k \Delta T$ and $1 \leqslant k \leqslant K$.

As pointed out in Section 1, post-prognostics scheduling strategies defined so far cannot be applied as is on fuel cells. In order to suit the schedule process to fuel cells specific characteristics and to their health state evolution over time, some properties simulating their behavior under wear and tear have to be taken into account. For each stack, available outputs and associated lifetimes are determined using a fuel cell behavior representation described in Section 3.

\section{Basic fuel cell wear and tear behavior}

Each fuel cell lifetime depends on both its current power output and its previous use, in addition to environmental and operating conditions, cell design and assembly and degradation mechanisms [1]. Even if operating conditions are known to have an impact on fuel cells durability, the consideration of many operating conditions during testing and data collecting is not yet mastered and needs further research [10]. Up to now, the impact of variable operating conditions on fuel cell lifetime is then not well-known and prognostics methods are not consistent with dynamic conditions. A simplified model depicting the behavior of fuel cells suffering from wear and tear and used with variable oper- 
ating conditions is then proposed in this section for PEMFC. This model can be used to determine at each time the available power outputs and their associated $R U L$. This representation is not an aging model that can be used for health assessment or $R U L$ prediction. In fact, it does not comply with all the fuel cell physical characteristics. However, it respects fuel cell behaviors which are useful to suit the scheduling process to fuel cell systems. Connection between the real behavior of fuel cells and the representation used in this paper for the scheduling process is shown in Figure 2.
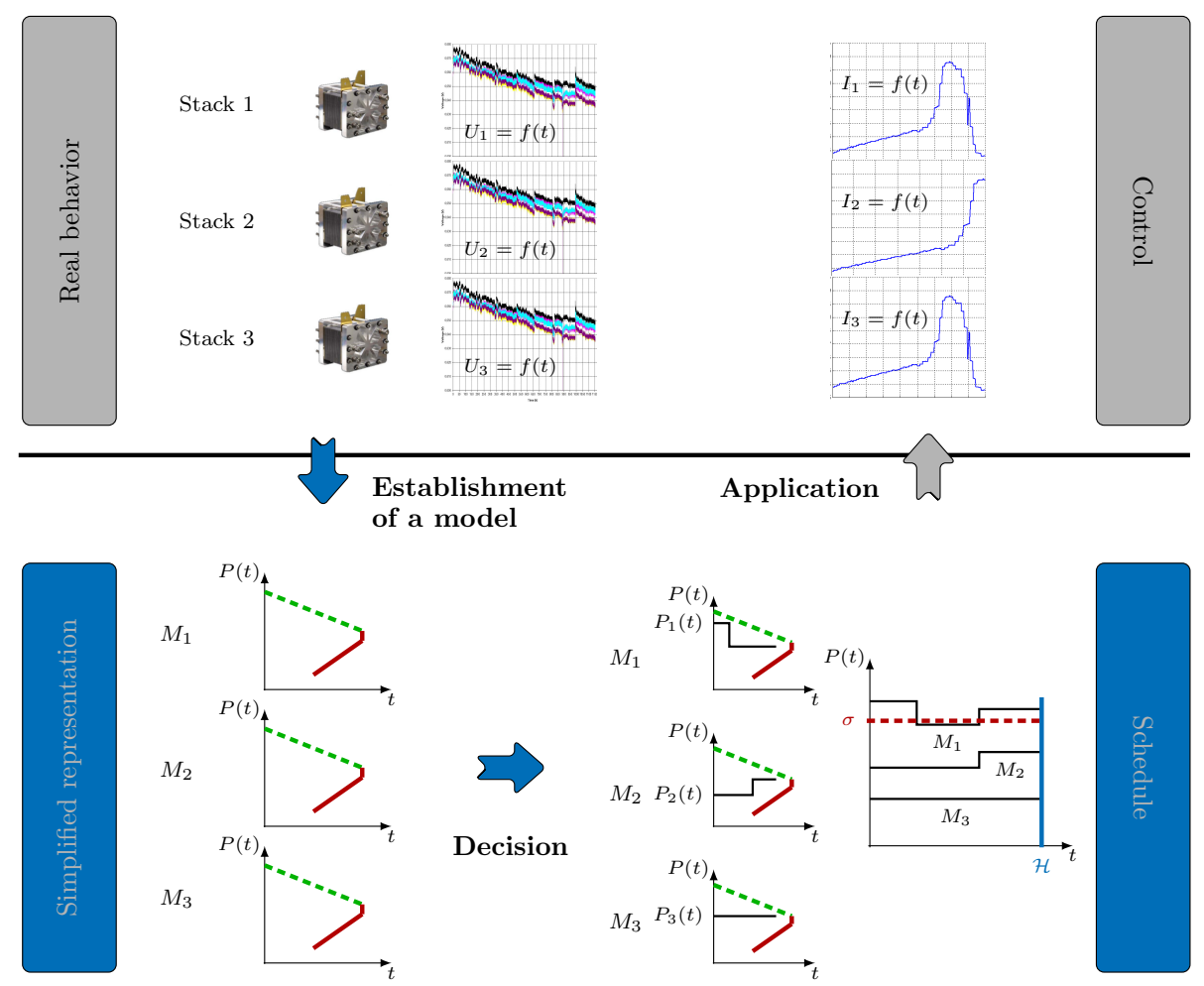

Figure 2: Connection between real behavior of fuel cells and its representation used for the scheduling process $-U_{j}$ : stack voltage, $I_{j}$ : stack current solicitation

\subsection{Health state prognostics model}

The proposed representation allows to determine the range of outputs available for a fuel cell stack $M_{j}$ throughout its use. This health state prognostics model has been based on the shape of the solid blue curve in Figure 3 which shows the typical evolution of the estimated $R U L$ in function of power for PEMFC. Proposed model illustrated in Figure 4 depicts the behavior of PEM fuel cells over time regarding their wear and tear. Some particular values can first be defined:

- $P \max _{j}$ is the maximal output, typically decreasing with time. The straight line depicting its decrease has equation $P \max _{j}(t)=a_{j} t+P \max _{j}(0)$, with $a_{j}$ the speed of the output decline and $P \max _{j}(0)$ the maximal output 
available at the beginning of the scheduling process. Both coefficients of this equation are fuel cell intrinsic characteristics ;

- $P \min _{j}$ is the minimal output, constant over time and defined as a percentage of the maximal one: $P \min _{j}=0.15 \cdot P \max _{j}(0)$. For outputs below this value, due to high voltage at the stack terminals, wear and tear is supposed to be too constraining to be consistent with an effective use. $P \min _{j}$ is associated to the minimal stack lifetime: $R U L_{j}\left(P \min _{j}\right)=$ $\min _{P \min _{j} \leqslant P_{j} \leqslant P \max _{j}} R U L_{j}\left(P_{j}\right)=R U L \min _{j}$;

- $P \operatorname{nom}_{j}$ is the power level recommended by fuel cell manufacturers for a nominal use of fuel cells, defined as $P \operatorname{nom}_{j}=0.75 \cdot P \max _{j}(0)$;

- Popt $_{j}$ is the output inducing the minimal wear and tear and associated to the maximal $R U L$. It corresponds to an optimal output considering fuel cell lifetime maximization as objective. This optimal output value being unknown, $P \operatorname{Popt}_{j}$ is approximated by $0.8 \cdot \operatorname{Pnom}_{j}$, which leads to $\operatorname{Popt}_{j}=0.6 \cdot \operatorname{Pmax}_{j}(0)$;

- $R U L \operatorname{opt}_{j}=R U L_{j}\left(P_{\mathrm{opt}_{j}}\right)$ is the maximal lifetime for the stack $M_{j}$, supposed to be reached by a subset of power outputs defined as follows: 0.9 opt $_{j} \leqslant P_{j} \leqslant \operatorname{Popt}_{j}$ (see Figure 3 ).

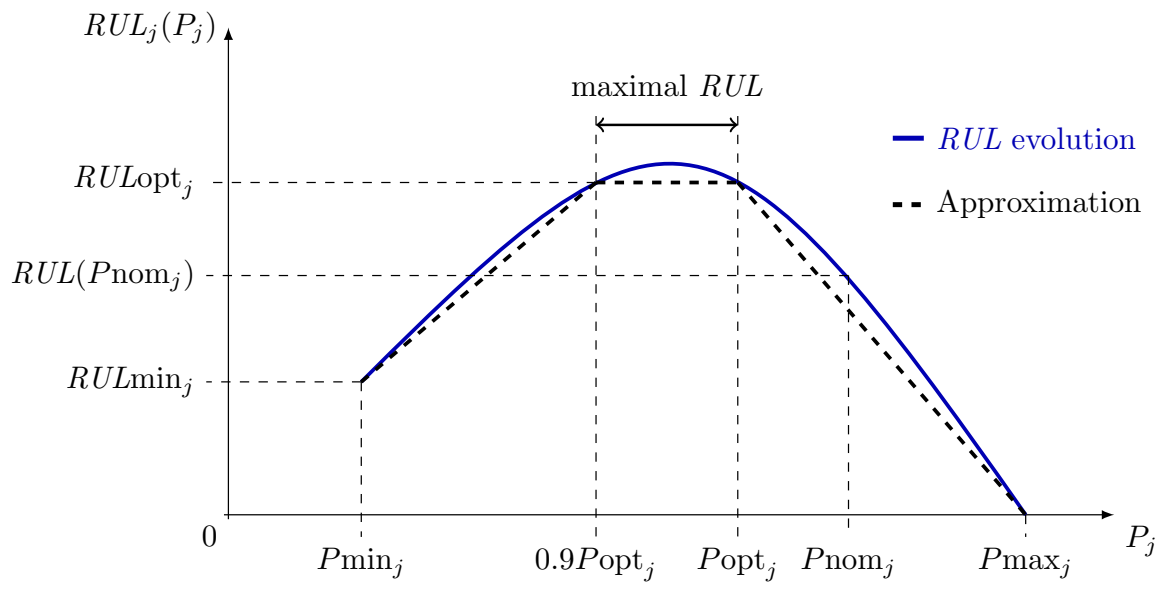

Figure 3: Approximation of the $R U L$ evolution of a fuel cell stack $M_{j}$ with power output

Associated $R U L$ for each output can be deduced from the representation in Figure 4 , in which bold lines represent the availability limit for outputs $P_{j}$ between $P \min _{j}$ and $P \max _{j}$. Three particular cases and two different behaviors regarding stack end of life can be distinguished:

- $P_{\text {opt }} \leqslant P_{j} \leqslant P \max _{j}: P_{j}$ useful life is limited by the decrease of $P \max _{j}$ of equation $P \max _{j}(t)=a_{j} t+b_{j}$, with $b_{j}=P \max _{j}(0)$, and depicted by a dashed green line in Figure 4 . In this output range, even if $P_{j}$ is not available anymore, the stack can still be used with lower outputs ;

In this first case, the limit represented by the dashed green line stands for a partial $R U L$, associated to a particular power output value. Reaching this value does not mean that the stack cannot be used anymore. For outputs $P_{j} \leqslant P_{\text {opt }_{j}}$, the limit represented by a solid red line in Figure 4 defines a global $R U L$ corresponding to the stack end of life. 


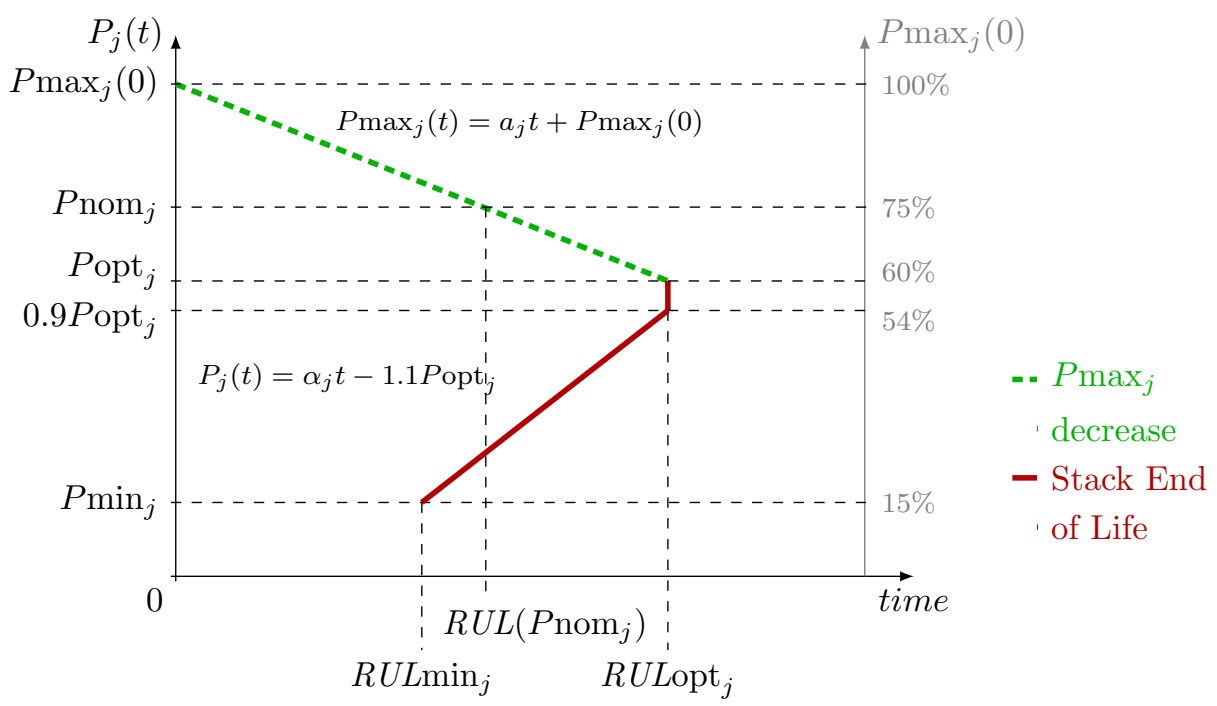

Figure 4: Characteristics evolution of a PEM fuel cell stack $M_{j}$ with time

- $0.9 \operatorname{Popt}_{j} \leqslant P_{j} \leqslant \operatorname{Popt}_{j}: P_{j}$ useful life is supposed to be maximal and to reach $R U L \operatorname{Lopt}_{j}$;

- $P \min _{j} \leqslant P_{j} \leqslant 0.9 P$ opt $_{j}: P_{j}$ useful life is penalized by the cut depicted by line of equation $P(t)=\alpha_{j} t+\beta_{j}$, with $\beta_{j}=-1.1 P \mathrm{opt}_{j}$. In fact, low outputs are associated to a greater wear and tear than $P_{\text {opt }}$.

All these properties taken into account in the health state prognostics model allow to fit it to fuel cell characteristics which are important for the addressed scheduling application.

\subsection{Compliance with fuel cell characteristics}

The model developed in previous section does not observe precisely the real characteristics of fuel cells. It respects however most important fuel cell behavior over time and particularly the influence of the past stack usage on its health state. The impact is different whether the outputs are chosen in the upper part of the model $\left(P \mathrm{opt}_{j} \leqslant P_{j} \leqslant P \max _{j}\right)$ or in the bottom part $\left(P \min _{j} \leqslant\right.$ $\left.P_{j} \leqslant P \mathrm{opt}_{j}\right)$. Compliance with an asymmetric behavior is first showed to be respected for power outputs greater than $\mathrm{Popt}_{j}$. A time equivalence is then proposed to comply with an appropriate health state evolution when a sequence of power outputs lower than $\mathrm{Popt}_{j}$ is defined in the schedule.

\subsubsection{Asymmetric behavior for $P$ opt $t_{j} \leqslant P_{j} \leqslant P \max _{j}$}

The asymmetric behavior regarding the outputs when $P_{j} \geqslant P$ opt $_{j}$ is well observed. In a schedule defining the contribution over time of a fuel cell stack, two successive outputs cannot always be permuted. Availability of outputs is in fact function of the past stack usage. As illustrated in Figure 5, the horizon reached by a stack depends on the output order over time. If the considered stack is first used with a high output, $P_{1}$, during a time $t_{1}$ and then with a smaller one, 
$P_{2}$, during a time $t_{2}$, the associated horizon is named $H\left(P_{1}, P_{2}\right)$. If the output order is reversed, $P_{2}$ can still be used during $t_{2}$, but after this, availability of $P_{1}$ is not enough to allow its use during $t_{1}$. Then, $H\left(P_{2}, P_{1}\right)<H(P 1, P 2)$.

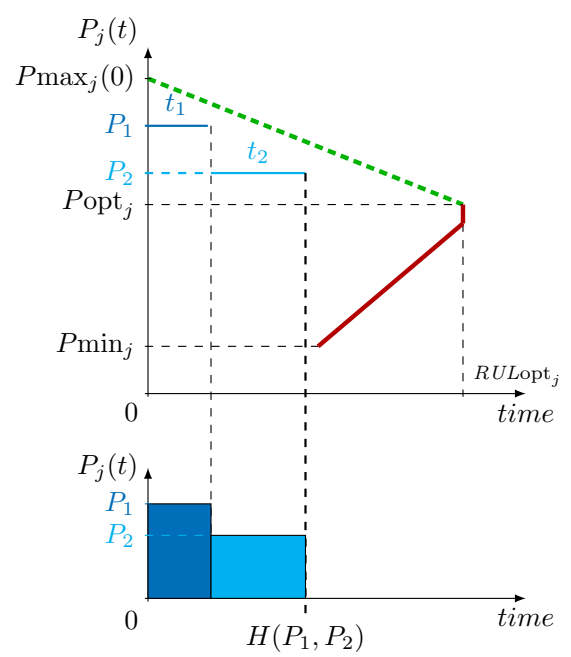

(a) First scenario: $P_{1}$ during $t_{1}$, then $P_{2}$ during $t_{2}$

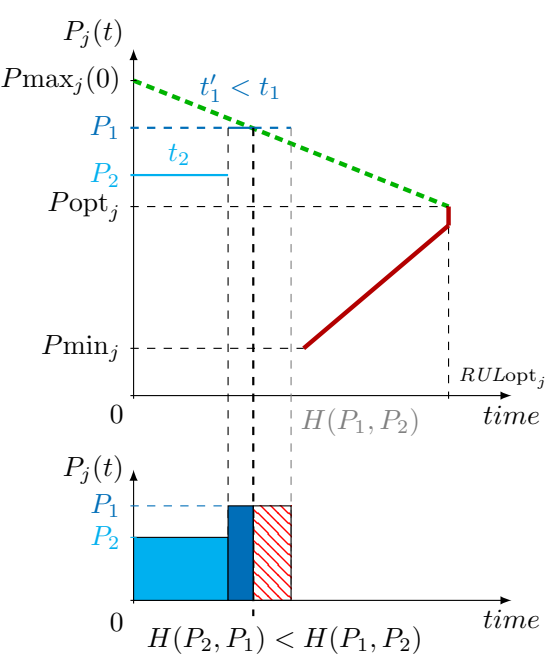

(b) Second scenario: $P_{2}$ during $t_{2}$ then $P_{1}$ during $t_{1}^{\prime}$

Figure 5: Illustration of the asymmetric property of fuel cell behavior for $P \operatorname{opt}_{j} \leqslant P_{j} \leqslant P \max _{j}$

\subsubsection{Time equivalence for $P \min _{j} \leqslant P_{j} \leqslant P$ opt $_{j}$}

For power outputs in the bottom part of the model $\left(P \min _{j} \leqslant P_{j} \leqslant P \mathrm{opt}_{j}\right)$, reaching of the limit represented by a line of equation $P(t)=\alpha_{j} t-1.1 P$ opt $_{j}$ (see the solid red line in Figure 4) corresponds to the stack end of life. A time equivalence is defined to comply with the fact that using a power output $P_{1}$ during a time $t_{1}$ does not involve the same wear and tear than using an other power output $P_{2}$ during the same time $t_{1}$. The point is then to define a quantity that can be used to compare the state of health of a stack after the use of any power output during a certain time. This quantity, named $X$, is defined as follows: considering an initial value $X=0$ (corresponding to a wear and tear of $0 \%)$, if the stack is used with an output $P_{1}$ during a time $t_{1}$, then the reached wear and tear is $X=t_{1} / R U L\left(P_{1}\right)$. After the use of $P_{1}$, if the stack has to be used with an other output $P_{2}, X$ allows to find the time associated to the wear and tear that would have been reached if the stack had been used with $P_{2}$ during $t_{1}$. This time is defined by: $t_{1,2}=X \times R U L\left(P_{2}\right)=t_{1} R U L\left(P_{2}\right) / R U L\left(P_{1}\right) \cdot t_{1,2}$ is the time at which the use of the stack with $P_{2}$ will begin after an use of $P_{1}$ during $t_{1}$ (see Figure 6).

Equivalent times can be found for all the power outputs following the straight line of equation $P_{j}(t)=\alpha_{j}^{\prime} t+\beta_{j}^{\prime}$, with $\alpha_{j}^{\prime}=\alpha_{j} / X$ (see Figure 6). 


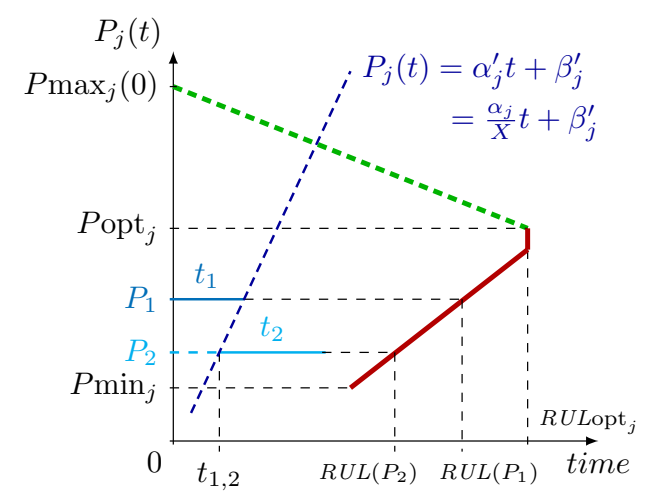

Figure 6: Illustration of the time equivalence for $P \min _{j} \leqslant P_{j} \leqslant P \mathrm{opt}_{j}$

\section{Resolution}

Proposed resolution method makes use of many successive sub-problem resolutions to cope with the decision problem defined in previous section. A Mixed Integer Linear Programming (MILP) approach, detailed in Section 4.1, is used to find the optimal assignment of stacks considering the initial health state of fuel cells, the load demand and the objective.

Horizons of solutions obtained with each MILP are limited either by the minimal $R U L$ of selected stacks with selected outputs or by the time during which the demand remains constant. Supposing that all the stacks are not necessary to reach the demand or that the limiting stack can still be used with a lower output, many $M I L P$ may be launched one after the other until the system remaining maximal global output gets below the demand. As the change of output during each stack lifetime is not managed by the MILP approach, a global resolution, proposed in Section 4.2, is needed to exploit all the system potential.

\subsection{Mixed Integer Linear Programming approach}

A mathematical program is used to find the optimal subset of fuel cell stacks and their configuration to reach a constant demand $\sigma$ during the maximal amount of time. Let $x_{j}(1 \leqslant j \leqslant m)$ be the binary decision variables such that $x_{j}=1$ if fuel cell stack $M_{j}$ is used in the solution and $x_{j}=0$ otherwise. Let $P_{j}$ $(1 \leqslant j \leqslant m)$ be the real variables defining each stack contribution to the global output. Values taken by $P_{j}(1 \leqslant j \leqslant m)$ are constrained between a minimal output, $P \min _{j}$, and a maximal one, $P \max _{j}$. These bounds express the range of available outputs for each fuel cell stack.

Main part of the objective function expressed in Equation (1) is the maximization of the production horizon $H$. For each solution, this horizon is limited by the minimal $R U L$ of selected stacks with selected power outputs: $H=\min _{j \mid x_{j}=1}\left(R U L_{j}\right)$. This corresponds to a max-min problem. A particular constraint is then defined in Equation (2) to limit the production horizon 
depending on the mathematical program solution.

$$
\begin{aligned}
& \max \quad H-\lambda\left(\sum_{j=1}^{m} x_{j} \cdot P_{j}-\sigma\right) \\
& H \leqslant R U L_{j} \quad \forall j=1, \ldots, m
\end{aligned}
$$

Second part of the objective function defined in Equation (1) is the overproduction minimization. No storage being made, restriction of the overproduction allows to minimize losses, which can be due either to wasted power or to an excessive need in chemical reactants. It results also in the minimization of the number of stacks used simultaneously to reach the load demand. This allows to maximize the global remaining potential of the set of stacks and may increase the global production horizon. This secondary objective is weighted with a multiplying factor, $\lambda$, allowing to grant it more or less importance.

Some constraints have to be taken into account in the linear program to comply with fuel cell intrinsic characteristics and requirements linked with the decision problem considered. Limited availability of each power output over time is expressed by Equations (3), (4) and (5). Each of these sets of constraints corresponds to one part of the RUL limitation illustrated in Figure 4. Equation (3) stands for the $P \max _{j}$ decrease depicted by a dashed green line in Figure 4 and applies for power outputs $P_{j} \geqslant P_{\text {opt }}$. Equation (4) applies for $0.9 \mathrm{Popt}_{j} \leqslant P_{j} \leqslant \mathrm{Popt}_{j}$ and Equation (5) for $P_{j} \leqslant 0.9 \mathrm{Popt}_{j}$. They stand for the stack end of life depicted by a red line in Figure 4. The shape of the $R U L$ evolution as a function of power output being concave (see Figure 3) and the three equations defining upper bounds for $R U L$ values, the simultaneous consideration of these equations allows to link the output value $P_{j}$ with the appropriate $R U L_{j}$. The term $C\left(1-x_{j}\right)$ has been added to arbitrarily overestimate the $R U L$ of machines that are not used in the solution (when $x_{j}=0$ ), with $C>\max _{j}\left(R U L \operatorname{Lpt}_{j}\right)$, so that the solution horizon $H$ is not constrained by the $R U L$ of a stack which is not used (see Equation (2)).

$$
\begin{array}{ll}
0 \leqslant R U L_{j} \leqslant \frac{P_{j}-b_{j}}{a_{j}} x_{j}+C\left(1-x_{j}\right) & \forall j=1, \ldots, m \\
0 \leqslant R U L_{j} \leqslant R U L \operatorname{sopt}_{j} . x_{j}+C\left(1-x_{j}\right) & \forall j=1, \ldots, m \\
0 \leqslant R U L_{j} \leqslant \frac{P_{j}-\beta_{j}}{\alpha_{j}} x_{j}+C\left(1-x_{j}\right) & \forall j=1, \ldots, m
\end{array}
$$

Reaching of the target global output value $\sigma$ is finally ensured by the constraint expressed in Equation (6).

$$
\sum_{j=1}^{m} x_{j} \cdot P_{j} \geqslant \sigma
$$

The mathematical program defined by the previous equations for a constant load demand $(\sigma(t)=\sigma)$ can be used to cope with a load demand piecewise constant over time $\left(\sigma(t)=\sigma_{k}\right)$. Solutions provided by this program could simply be truncated to the time during which the demand remains constant, but a better assignment of stacks may be found for a shorter horizon. In order to find the optimal assignment for the considered load demand $\sigma_{k}$ during the 
associated limited time $t_{\max }=t_{k}$, an additional constraint limiting the solution horizon $H$ can be considered as defined in Equation (7).

$$
H \leqslant t \max
$$

When the load demand is constant during the whole production horizon, tmax is overestimated such that $t \max >\max _{j}\left(R U L o p t_{j}\right)$. This allows to find the solution having the maximal horizon whatever the situation, that is whether the solution horizon is limited by the $R U L$ of a limiting stack or by the time during which the load demand remains constant. Considering this, the objective function remains the same for a piecewise constant demand, as well as the sets of constraints previously defined.

Linear optimization is proposed to cope with the maximization problem. The term $x_{j} . P_{j}$ being the product of a binary variable and a real one, the mathematical program defined by the previous equations is not linear. Linearization is then mandatory and is made following the properties defined in set of Equations (8).

$$
\begin{aligned}
\forall x \in\{0,1\}, \forall y \in[0, U(y)] \text { and } \forall e \in \mathbb{R}, e=x y \text { if and only if: } \\
\qquad\left\{\begin{array}{l}
e \leqslant x U(y) \\
e \leqslant y \\
e \geqslant y-(1-x) U(y) \\
e \geqslant 0
\end{array}\right.
\end{aligned}
$$

The linearized formulation of the Mixed Integer Linear Program is given in set of Equations (9), with $e_{j}=x_{j} . P_{j} \forall j=1, \ldots, m$. This $M I L P$ allows to find an optimal configuration, i.e., an optimal power output value for each available fuel cell stack, that maximizes the solution horizon. This program can be used whether the demand is constant over time or piecewise constant. 


$$
\begin{cases}\max & H-\lambda\left(\sum_{j=1}^{m} e_{j}-\sigma\right) \\ & H \leqslant R U L_{j} \quad \forall j=1, \ldots, m \\ & H \leqslant t \max \\ & \sum_{j=1}^{m} e_{j} \geqslant \sigma \\ & e_{j} \leqslant x_{j} P \max _{j} \quad \forall j=1, \ldots, m \\ \text { s.t. } & e_{j} \leqslant P_{j} \quad \forall j=1, \ldots, m \\ & e_{j} \geqslant P_{j}-\left(1-x_{j}\right) P \max _{j} \quad \forall j=1, \ldots, m \\ & e_{j} \geqslant 0 \quad \forall j=1, \ldots, m \\ & 0 \leqslant R U L_{j} \leqslant \frac{e_{j}}{a_{j}}-\frac{b_{j}}{a_{j}} \cdot x_{j}+C\left(1-x_{j}\right) \quad \forall j=1, \ldots, m \\ & 0 \leqslant R U L_{j} \leqslant R U L \mathrm{opt}_{j} \cdot x_{j}+C\left(1-x_{j}\right) \quad \forall j=1, \ldots, m \\ & 0 \leqslant R U L_{j} \leqslant \frac{e_{j}}{\alpha_{j}}-\frac{\beta_{j}}{\alpha_{j}} \cdot x_{j}+C\left(1-x_{j}\right) \quad \forall j=1, \ldots, m \\ \text { with } & P_{j} \geqslant P \min _{j} \geqslant 0 \quad \forall j=1, \ldots, m \\ & 0 \leqslant P_{j} \leqslant P \max _{j} \quad \forall j=1, \ldots, m \\ & x_{j} \in\{0,1\} \quad \forall j=1, \ldots, m\end{cases}
$$

\subsection{Resolution algorithm}

Succession of MILP and update of fuel cell stacks characteristics between each launch of Linear Program is detailed in Algorithm 1. In comparison with a constant load demand, the load value taken into account by the linear program, as well as the associated duration, change over time when considering a variable demand. In this case, an additional step has to be performed to update these values during the resolution process.

Global lifetime of the multi-stacks fuel cell system, $\mathcal{H}$, corresponds to the sum of horizons reached by all the successive MILP solutions. Solutions provided by each Linear Program are optimal considering the health state of stacks at the beginning of each MILP, but global schedules obtained with the succession of many MILP are not necessarily optimal.

\section{Simulation results}

Simulations have been conducted to evaluate the approach proposed in the previous section. After a description of the problem generation, efficiency of the approach is assessed for a constant load demand as well as for a demand piecewise constant over time. This is consistent with the considered application framework, in which PEM fuel cell systems are used to meet energy requirements for stationary applications (e.g. domestic usage).

When considering a constant load demand profile, the strategy proposed in Section 4 for the management of fuel cell stacks is compared to basic strate- 


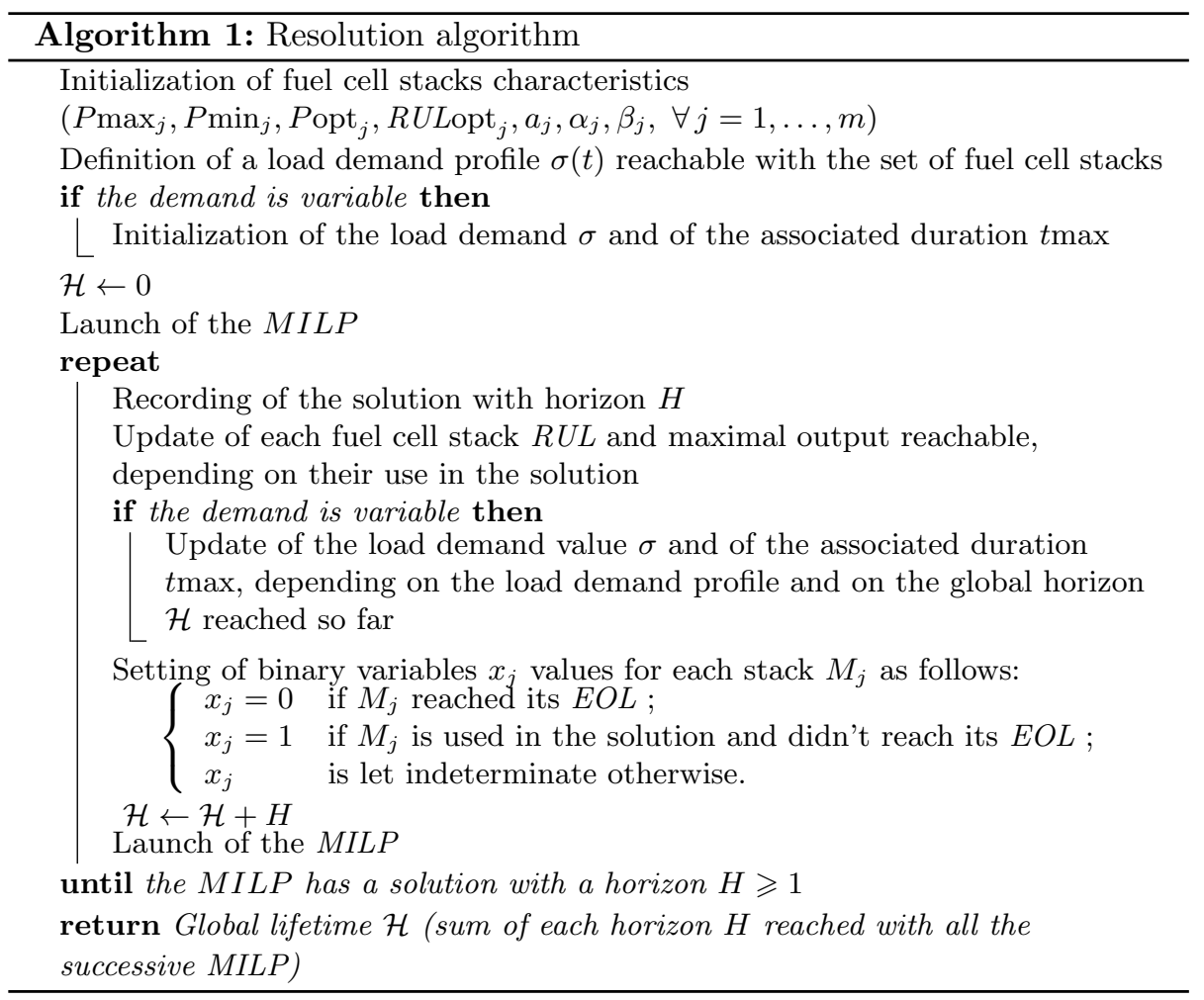

gies defining intuitive rules for the choice of output values. Robustness of the approach is then assessed on variable load demand profiles.

\subsection{Problem generation}

Random problem configurations have been generated using a simulator and configured with parameters such as the number of fuel cell stacks considered, $m$, and intrinsic characteristics of PEMFC (maximal output and its decrease rate, minimal output, etc. - see Figure 4 -). These latter characteristics have been defined on the basis of fuel cell manufacturer specifications and studies conducted to evaluate fuel cells behavior under wear and tear. Power values taken into account for simulations are the following for each stack $M_{j}: \operatorname{Pmax}_{j}(0)=$ $500 \mathrm{~W} \pm 5 \% ; P \min _{j}=0.15 \cdot P \max _{j}(0) ; P \mathrm{opt}_{j}=0.8 \cdot P \operatorname{nom}_{j}=0.6 \cdot P \max _{j}(0)$. A multi-stacks fuel cell system may include stacks with same output characteristics, but these can be differentiated by their $R U L$ set by the slope depicting the maximal output decrease over time $\left(a_{j}\right)$. It is indeed assumed that stacks can have different states of health at the beginning of the scheduling process. Values for each slope $a_{j}$ have been determined considering a maximal lifetime $R U L$ opt $_{j}=1500$ hours $\pm 20 \%$ for each stack. Then, $a_{j} \in[-0.08,-0.13] W . h^{-1}$.

When considering a load demand constant over time, only one demand value $\sigma$ has been associated to each problem configuration, but many demands corresponding to different configurations have been tested. Many loads $\delta$ have been defined such that $\sigma=\delta \cdot P$ nom $_{t o t}$, with $P$ nom $_{t o t}=\sum_{j=1}^{m} P$ nom $_{j}$, the nominal total output reachable with the considered set of fuel cell stacks and 
$30 \% \leqslant \delta \leqslant 90 \%$. Each result is the average of 20 random instances corresponding to one problem configuration as defined before. In the following figures, results are represented as a function of the load $\delta$.

Robustness of the commitment strategy is then proposed to be assessed on a variable load demand profile. The variable demand being supposed to be piecewise constant for the considered micro-CHP context, the demand profile is divided into $k$ time periods, each of them being associated to a certain load demand value $\sigma_{k}$. The instant demand $\sigma_{k}$ is supposed to fluctuate over time around a mean load demand value $\sigma_{\text {mean }}$. As for the case considering a constant load demand, many instances of the decision problem are considered, with $\sigma_{\text {mean }}=\delta \cdot$ Pnom $_{t o t}$ and $30 \% \leqslant \delta \leqslant 90 \%$. For each problem instance, $\sigma_{k}$ is supposed to take successively a minimal value, $\sigma_{\min }=\sigma_{\text {mean }}-\Delta \sigma$, and a maximal one, $\sigma_{\max }=\sigma_{\text {mean }}+\Delta \sigma$ (see Figure 7$)$. Two standard load demand profiles are considered. First one begins with the minimal demand value, $\sigma_{\min }$ (see Figure $7 \mathrm{a}$ ). Second one begins with the maximal value, $\sigma_{\max }$ (see Figure $7 \mathrm{~b}$ ).

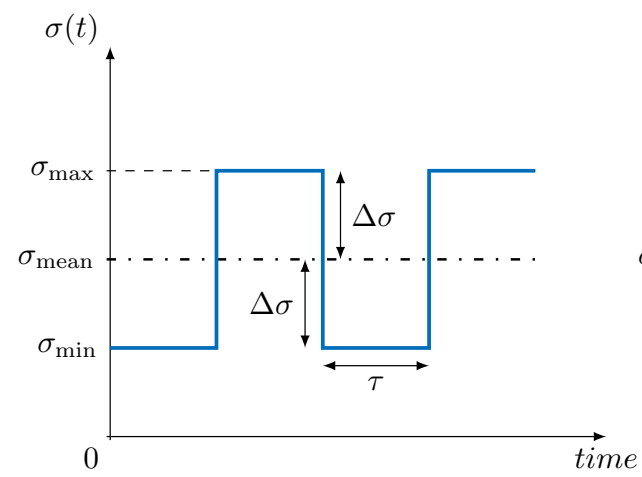

(a) Demand profile type 1

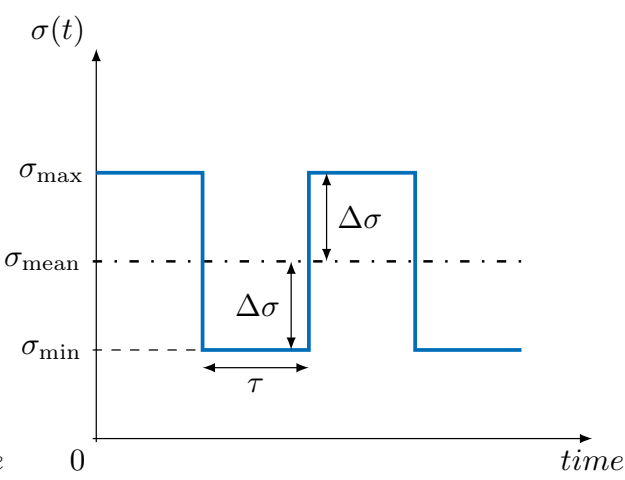

(b) Demand profile type 2

Figure 7: Variable load profiles considered for simulations

\subsection{Results}

Results do not vary significantly with the number of stacks considered. For the results proposed hereafter, the parameter $m$ has been set to 20 . This value is consistent with the considered stationary application. Production horizons reached by solutions provided by each MILP have been rounded down to the lower integer. Supposing that $R U L$ values are given in hours or days units, this avoids applying solutions to fractions of these units. Parameter $\lambda$ in the objective function has been set to 2 for each MILP. This value allows to avoid overproduction and to reach greater production horizons than with $\lambda<2$. For readability reasons, points associated to the different results shown in following figures have been scattered around the corresponding load value on the abscissa.

\subsubsection{Constant load demand}

Efficiency of the proposed commitment strategy defined in Section 4, named $S 1$, is first assessed through a comparison with two basic intuitive strategies, considering a load demand constant over time during the whole production horizon: 
$\sigma(t)=\sigma$. First one, $S 2$, conforms to fuel cell manufacturers recommendations and constraints the use of stacks at their nominal output $P$ nom $_{j}$ during their whole lifetime. This strategy corresponds to a classical usage of fuel cell systems. Strategy $S 3$ limits the selection of power output in the range associated to the maximal $R U L,\left[0.9 \mathrm{Popt}_{j}, \mathrm{Popt}_{j}\right]$ (see Figure 4). For all the strategies, a continuous use of fuel cell stacks is observed. In accordance with the assumption detailed in Section 2.1, no stop-and-start is then allowed during the scheduling process.

Figure 8 shows the production horizons (in hours) reached with all the considered strategies as a function of the load $\delta$. Evolution of an upper bound for the production horizon is also depicted. Considering a constant demand $\sigma$ and a set of fuel cell stacks, this upper bound, denoted UB and defined in Equation (10), corresponds to the theoretical maximal time during which the load demand can be reached. It can be geometrically defined as the sum for each stack of the surface below the curve depicting the maximal output decrease with time, divided by the load demand. As this simplified expression does not take into account the lifetime cut-off defined in the MILP formulation by Equation $(9 \mathrm{k})$ for power outputs $P_{j} \leqslant 0.9 P_{\text {opt }}$, this upper bound is never reachable.

$$
\mathrm{UB}=\left\lfloor\sum_{j=1}^{m} \frac{1}{2}\left(1.6 P \max _{j}-2 P \min _{j}\right) \cdot R U L \mathrm{opt}_{j} / \sigma\right\rfloor
$$

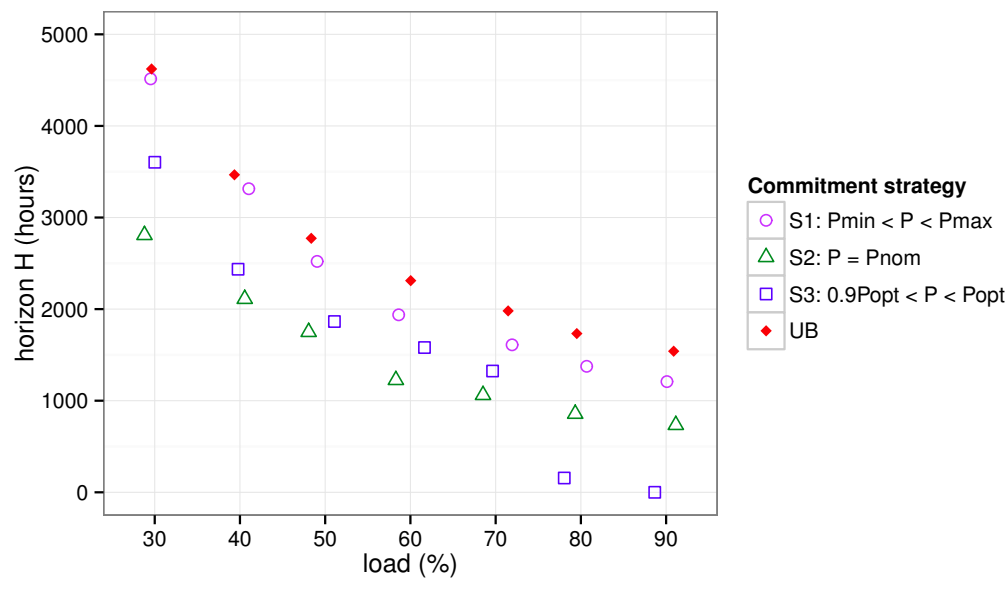

Figure 8: Comparison of production horizons reached with several commitment strategies

One can see in Figure 8 that strategy $S 2$, setting each fuel cell contribution to its nominal output, decreases with the load $\delta$. No power output choice is performed and lifetime of each stack $M_{j}$ is limited by the lifetime associated to $P$ nom $_{j}$. Only the number of parallel machines needed to reach the demand $\sigma$ varies in function of the load. When the load increases, the number of machines needed in each period of time increases. Less group of parallel machines can then be constituted, which implies a decrease of the horizon $\mathcal{H}$. Strategy 
$S 3$, limiting the selection of outputs in the range $\left[0.9 \mathrm{Popt}_{j}, \mathrm{Popt}_{j}\right]$, is more efficient, but not reliable for high loads. The tested demands $\sigma$ being defined as a percentage of the nominal total output reachable with the considered set of fuel cell stacks, $P$ nom $_{t o t}$, and knowing that $P \mathrm{opt}_{j}=0.8 \cdot P \mathrm{nom}_{j}$ for each stack $M_{j}$, a load $\delta=90 \%$ is not reachable with strategy $S 3$. A load $\delta=80 \%$ is also not always reachable, due to rounding of numbers for the determination of $P$ nom $_{\text {tot }}$. Greater production horizons are reached with strategy $S 1$. Results provided by this strategy being not so far from the upper bound, they are close to the optimal.

Same behavior can be observed in Figure 9, which shows the distance of production horizons obtained with all the considered strategies to the theoretical upper bound, UB, defined in Equation (10). This upper bound being not reachable, results are actually better than showed in this Figure. Strategy $S 2$ allows to reach a mean relative horizon of around $55 \%$. With strategy $S 3$, this mean relative horizon reaches $51 \%$, but is close to $0 \%$ for high loads. Best results are obtained with the strategy $S 1$, which allows to reach relative horizons between $78 \%$ and $97 \%$ of the upper bound UB and $86 \%$ on average.

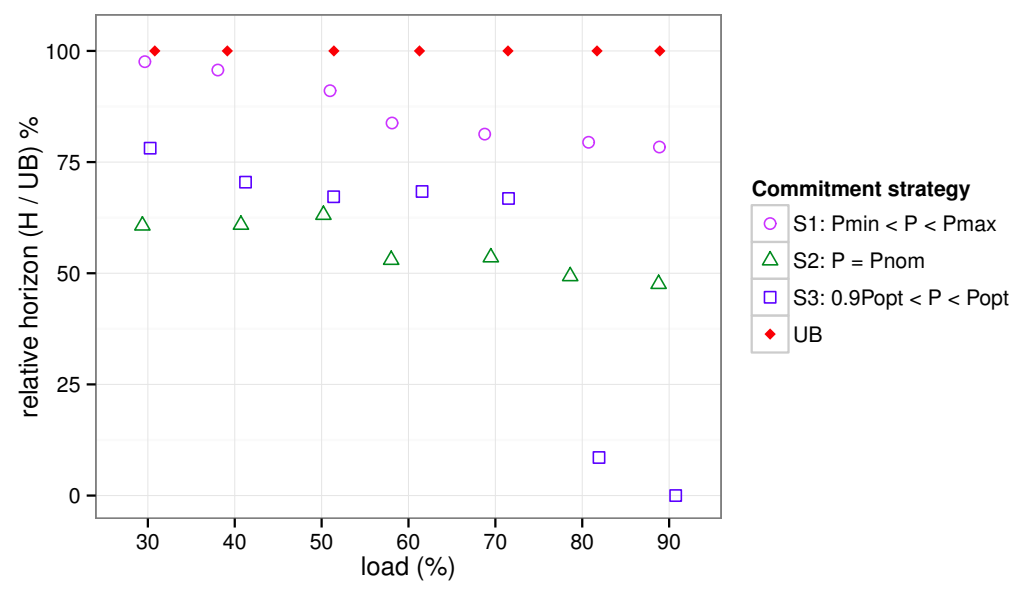

Figure 9: Distance to a theoretical upper bound of production horizons reached with several commitment strategies

When considering a constant load demand, the proposed strategy $S 1$ allowing to choose power outputs in the whole available output range $\left[P \min _{j}\right.$, $\left.P \max _{j}(t)\right]$ allows then to extend the multi-stacks fuel cell system lifetime in comparison with basic strategies limiting the output range.

Computation time ${ }^{1}$ increases with the load and with the number of machines, but solutions are found in less than 1 minute for all the tested configurations.

\subsubsection{Piecewise constant load demand}

Robustness of the strategy producing best results for a load demand constant over time, $S 1$, is assessed for variable load demand profiles, $\sigma(t)=\sigma_{k}$. For the

\footnotetext{
${ }^{1}$ Simulations have been launched using MATLAB ${ }^{\circledR}$ and the solver for linear programming Gurobi ${ }^{\circledR}$ (Computation parameters: Processor Intel Core ${ }^{T M}$ i5-3550 CPU 3.30GHz $\times 4,15.6$ Gio, 64 bits)
} 
results proposed hereafter, time periods are supposed to be of equal duration with $\tau=U B / k, U B$ being the upper bound for the production horizon and $k$ the number of periods considered (see Figure 7 ). As the mean load demand over the profile horizon equals $\sigma_{\text {mean }}$, the upper bound remains the same as the one defined in Equation (10) for a constant load demand. Load demand profiles have been divided into 10 time periods. Three different amplitudes for the demand variations $\Delta \sigma$ are compared. First one considers a variation rate of the load $\delta \pm 10 \%$, such that $\sigma_{\min }=0.9 \delta \cdot P_{n^{\prime}} m_{t o t}$ and $\sigma_{\max }=1.1 \delta \cdot P$ nom $_{\text {tot }}$. Second one considers $\delta \pm 20 \%$ and the third one $\delta \pm 30 \%$. As $\sigma_{\min }=0$ when considering $\delta-30 \%$ with $\delta=30 \%$, results for loads $\delta=30 \%$ are not represented in following figures. The strategy $S 1$, giving best results for a constant load demand, is used to compare results obtained with these three demand variations.

One can see in Figure 10 that efficiency of the commitment strategy decreases when the load variation rate increases, whatever the demand profile type considered. Reached production horizons decrease also when the load demand $\delta$ increases. Depending on the order of the different values taken by the load demand during the profile horizon, reached production horizons are not the same. In fact, different results are obtained with the profile type 1 which begins with a low demand value, $\sigma_{\min }$, and with a profile type 2 beginning with $\sigma_{\max }$. The global need in power output being different, a different number of stacks may be involved depending on the first part of the profile. Because a continuous use of stacks is observed once they have been started, decisions taken for the first part of the profile impact the following ones and, by extension, impact also the production horizon. This can be seen in Figure 10: for the same demand variation rate, production horizons reached with the standard profile type 2 are less than those reached with standard profile type 1 . Differences increase with the load demand variability. Anyway, results remain promising since they reach at least $71 \%$ (resp. $64 \%$ ) of the theoretical maximal horizon for a mean load demand $\delta=50 \%$ with profile type 1 (resp. profile type 2 ). The resolution method proposed for a constant load demand can then be used for piecewise constant demand profiles.

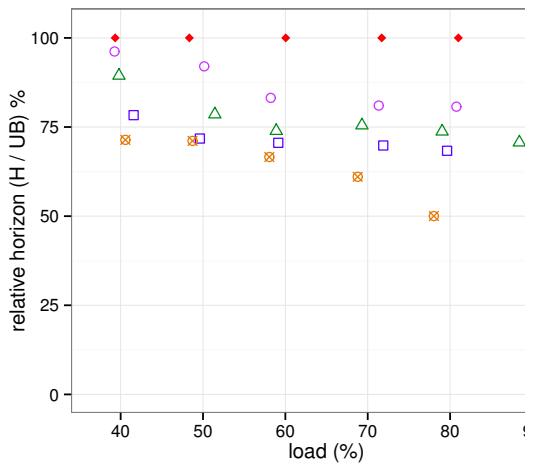

(a) Demand profile type 1

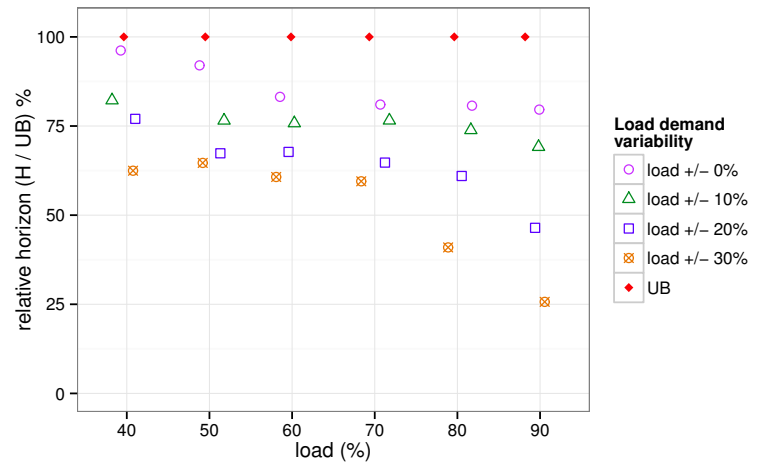

(b) Demand profile type 2

Figure 10: Distance to a theoretical upper bound of production horizons reached with commitment strategy $S 1$, considering variable load demands

Considering a piecewise constant load demand, computation time ${ }^{1}$ increases 
with the load and with the number of machines. As for a constant load demand, the method remains all the same time-efficient. In fact, solutions are found in less than 1 minute for all the tested configurations. The load demand being considered constant over time or piecewise constant, the proposed resolution method is then consistent with the mid-term decision level considered for the application (decision frequencies from hours to weeks).

\section{Conclusion}

A management of multi-stacks fuel cell systems has been proposed in a PHM framework. The maximization of such systems lifetime has been addressed using fuel cell stacks $R U L$ information. As the determination of such $R U L$ values still needs deep studying in the prognostics domain, a simplified representation of the fuel cell stacks behavior under wear and tear has been proposed. This health state prognostics model has been configured to suit to PEM Fuel Cells. The estimation of stacks available outputs and their associated $R U L$ evolution with time deduced from this representation has been used to define a scheduling of stacks in power. The proposed method, making use of successive optimal resolutions obtained with a mixed integer linear program, allows to extend a multi-stacks fuel cell system useful life under constant power need. In fact, the proposed strategy, which allows to choose power outputs in the whole output range $\left(\left[P \min _{j}, P \max _{j}\right]\right)$ for each stack, has been shown to be more efficient than a constant control of stacks with the nominal power output, $P$ nom, or with the one associated to the longest $R U L$, Popt. Comparison between strategies has been made considering a constant load demand. Robustness of the best strategy has then been assessed for variable load demands. Definition of considered piecewise load demand profiles has been based on a cyclic variation of the load between a minimal value and a maximal one, which is a typical characteristic of a micro-CHP application. Results show that the approach is consistent with a real usage of fuel cells in domestic applications.

A particular strategy can however be developed for variable load demand profiles to propose a better assignment of stacks and improve the reached production horizons. In fact, due to the constraint allowing no stop-and-start of stacks, all the stacks used to deal with a high load demand are still used when the load demand decreases if they did not reach their end of life. One can however imagine that less stacks would be enough to reach the lower load demand. Relaxation of the constraint imposing a non-interrupted use of stacks will be addressed. Some stop-and-start will then be allowed during the stacks use. Resulting damage to the cells will be taken into account through a certain $R U L$ decrease for affected stacks. Future work will also include the search for a better objective function for the $M I L P$ formulation. Enhancement of the model defining the available outputs over time and their associated $R U L$ will be studied as well by considering a quadratic function to depict the maximal output decrease with time. This will allow to suit even more the model to the real behavior of fuel cells. Reuse of overproduction will finally be addressed by the study of a storage management. 


\section{Acknowledgment}

This work has been supported by the Labex ACTION project (contract "ANR11-LABX-01-01")

\section{References}

[1] M. Jouin, R. Gouriveau, D. Hissel, M.-C. Péra, N. Zerhouni, Prognostics and health management of PEMFC - state of the art and remaining challenges, International Journal of Hydrogen Energy 38 (2013) 15307-15317.

[2] S. Sichilalu, H. Tazvinga, X. Xia, Optimal control of a fuel cell/wind/PV/grid hybrid system with thermal heat pump load, Solar Energy 135 (2016) 59-69.

[3] C. Azcárate, R. Blanco, F. Mallor, R. Garde, M. Aguado, Peaking strategies for the management of wind- $\mathrm{h}_{2}$ energy systems, Renewable Energy 47 (2012) 103-111.

[4] N. Bigdeli, Optimal management of hybrid pv/fuel cell/battery power system: A comparison of optimal hybrid approaches, Renewable and Sustainable Energy Reviews 42 (2015) 377-393.

[5] R. Dufo-López, J. L. Bernal-Agustín, J. Contreras, Optimization of control strategies for stand-alone renewable energy systems with hydrogen storage, Renewable Energy 32 (2007) 1102-1126.

[6] I. Abadlia, T. Bahi, H. Bouzeria, Energy management strategy based on fuzzy logic for compound RES/ESS used in stand-alone application, International Journal of Hydrogen Energy 41 (2016) 16705-16717.

[7] K. Rajashekara, Hybrid fuel-cell strategies for clean power generation, IEEE Transactions on Industry Applications 41 (3) (2005) 682-689.

[8] H. Aouzellag, K. Ghedamsi, D. Aouzellag, Energy management and fault tolerant control strategies for fuel cell/ultra-capacitor hybrid electric vehicles to enhance autonomy, efficiency and life time of the fuel cell system, International Journal of Hydrogen Energy 40 (2015) 7204-7213.

[9] A. Neffati, M. Guemri, S. Caux, M. Fadel, Energy management strategies for multi source systems, Electric Power Systems Research 102 (2013) 4249.

[10] R. Borup, J. Meyers, B. Pivovar, M. Inaba, et al., Scientific aspects of polymer electrolyte fuel cell durability and degradation, Chemical reviews 107 (10) (2007) 3904-3951.

[11] B. Saha, C. C. Quach, K. Goebel, Optimizing battery life for electric UAVs using a bayesian framework, in: Proc. of IEEE Aerospace Conference, Big Sky, MT, USA, 2012.

[12] A. D. Bosco, M. H. Fronk, Fuel cell flooding detection and correction, us patent (6.103.409), 2000. 
[13] B. Wells, R. K. Parr, Fuel cell system method, apparatus and scheduling, us patent (0185316 a1), 2004.

[14] N. Herr, J.-M. Nicod, C. Varnier, Prognostic decision making to extend a platform useful life under service constraint, in: Proc. of IEEE International Conference on Prognostics and Health management (PHM), Spokane, Washington, USA, 2014, Best paper Award.

[15] N. Herr, J.-M. Nicod, C. Varnier, Prognostics-based scheduling in a distributed platform: Model, complexity and resolution, in: Proc. of IEEE International Conference on Automation Science and Engineering (CASE), Taipei, Taiwan, 2014, pp. 1054-1059. 